\title{
Análise da vacina dupla adulto em gestantes de Santiago de los Caballeros.
}

\author{
Analysis of double vaccine adult in pregnant women of Santiago de los \\ Caballeros.
}

\section{Mirana Celipha Diometre ${ }^{1}$, Roberta Melo de Morais $^{2}$, Eliete Albano de Azevedo Guimarães ${ }^{3}$, Adriano Marçal Pimenta ${ }^{4}$, Gabriela Gonçalves Amaral ${ }^{5}$, Valéria Conceição de Oliveira ${ }^{6}$}

\begin{abstract}
${ }^{1}$ Mestre em Enfermagem. Universidade Federal de São João Del Rei (UFSJ), Programa de Pós-graduação em Enfermagem, Minas Gerais (MG), Brasil.
\end{abstract}

${ }^{2}$ Graduanda em Enfermagem. Universidade Federal de São João Del Rei (UFSJ), Curso de graduação em Enfermagem, Minas Gerais (MG), Brasil.

${ }^{3}$ Doutora em Enfermagem. Universidade Federal de São João Del Rei (UFSJ), Departamento de Enfermagem, Minas Gerais (MG), Brasil.

${ }^{4}$ Doutor em Enfermagem. Universidade Federal de Minas Gerais (UFMG), Escola de Enfermagem, Minas Gerais (MG), Brasil.

${ }^{5}$ Mestranda em Enfermagem. Universidade Federal de São João Del Rei (UFSJ), Programa de Pósgraduação em Enfermagem, Minas Gerais (MG), Brasil.

${ }^{6}$ Doutora em Enfermagem. Universidade Federal de São João Del Rei (UFSJ), Departamento de Enfermagem, Minas Gerais (MG), Brasil.

\section{Resumo}

Introdução: A vacinação é uma das medidas importantes durante a gravidez para impedir resultados adversos para a mulher e seu bebê através da imunidade ativa e passiva, respectivamente. Objetivo: Analisar o histórico da vacina dupla adulto (dT) e fatores relacionados em gestantes. Método: Estudo transversal realizado com uma amostra estimada de 741 puérperas de uma maternidade de Santiago de los Caballeros, República Dominicana em 2016. A variável desfecho do estudo foi a administração de dose de dT durante a gestação. Para avaliar os fatores associados, foi realizada a Regressão de Poisson Simples com variância robusta. Foram calculados os coeficientes de prevalência ajustados e seus respectivos intervalos de confiança de 95\%. Resultados: Participaram do estudo 726 puérperas. Uma proporção de $(57,3 \%)$ apresentava duas ou mais doses da vacina dT. O estado civil solteiro/separado/ divorciado, ter 2 ou mais filhos vivos e não receber indicações sobre a vacina durante o pré-natal foi negativamente relacionado ao desfecho, após o ajuste multivariado dos dados. Conclusões: Houve administração de duas ou mais doses de dT na maioria das gestantes. Isso pode estar relacionado à ausência do cartão de vacina, aumentando o risco de receber doses desnecessárias de vacinas durante o pré-natal.

Palavras-chave: Imunização; Vacina contra Difteria e Tétano; Mulheres grávidas; Enfermagem.

\section{Autora correspondente:}

Valéria Conceição de Oliveira

Endereço: Av.Sebastião Gonçalves Coelho, 400 - Chanadour,

CEP 35.504-296. Divinópolis (MG), Brasil.

Telefone: (37) 999853059

Recebido em: 06/05/2019

Revisado em: 14/06/2019

Aceito em: $12 / 07 / 2019$

E-mail: valeriaoliveira@ufsj.edu.br

Publicado em: 30/09/2019 


\section{Abstract}

Introduction: Vaccination is one of the main vitreous during pregnancy for adverse delivery for the woman and the baby through active and passive immunity, respectively. Objective: To analyze the history of the double adult vaccine $(d T)$ and related factors in pregnant women. Method: A cross-sectional study carried out with puerperal women at a maternity hospital in Santiago de los Caballeros, Dominican Republic. The outcome variable of the study was the administration of $d T$ dose during gestation. To evaluate the associated factors, the Simple Poisson Regression with robust variance was performed. The adjusted prevalence coefficients and their respective 95\% confidence intervals were calculated. Results: 726 postpartum women participated in the study. A proportion of (57.3\%) had two or more doses of the dT vaccine. Single / separated / divorced marital status, having 2 or more live children and no indication of the vaccine during prenatal care was negatively related to outcome after multivariate adjustment of data. Conclusion: There were two or more doses of $d T$ in the majority of pregnant women. This may be related to the absence of the vaccine card, increasing the risk of receiving unnecessary doses of vaccines during prenatal care.

\section{Keywords: Immunization; Diphtheria and Tetanus Vaccine; Pregnant women; Nursing.}

\section{Introdução}

A vacinação é uma das medidas importantes durante a gravidez para impedir resultados adversos para a mulher e seu bebê através da imunidade ativa e passiva, respectivamente ${ }^{1-2}$. Nos últimos anos, os dados acumulados mostraram benefícios e segurança de algumas vacinas para os bebês, quando administradas a suas mães, durante a gravidez ${ }^{3}$.

$\mathrm{Na}$ República Dominicana, o Programa Ampliado de Imunizações (PAI) iniciou suas atividades de prevenção de doenças no ano 1978 e, desde então, dispõe para a população adulta a vacina dupla adulto (dT), contra difteria e tétano, e não tem um sistema de registro informatizado de vacinas ${ }^{4}$. Esta vacina está indicada a partir dos sete anos de idade, para aqueles que receberam a vacina combinada contra a difteria, tétano e coqueluche (DTP), ou que tenham esquemas incompletos. Esta vacina é também recomendada pelo PAI para seu uso em grávidas e em mulheres em idade fértil (10 a 39 anos) para a prevenção de difteria e tétano nas mães e o tétano neonatal em seus filhos ${ }^{4}$. Recomenda-se continuar o esquema de vacinação com dT, iniciado em oportunidades anteriores ou gravidez, a fim de completar as doses necessárias. Apesar do Comitê Assessor sobre Práticas de Imunização (ACIP), desde outubro de 2011, recomendar a administração rotineira de uma dose de tétano, de difteria e coqueluche (dTpa) durante a gravidez, em um esforço para reduzir a coqueluche em lactantes ${ }^{5-7}$, na República Dominicana, esta recomendação ainda não foi incluída no calendário de vacinação das gestantes que normalmente são vacinadas com a vacina dupla adulto.

Na maioria dos países, inclusive na República Dominicana, recomenda-se uma série de três doses de vacina contendo o toxoide tetânico na infância, seguida de dose de reforço no segundo ano, outra em idade escolar e a cada dez anos ${ }^{4,8-9}$. Para as mulheres grávidas que nunca receberam vacinas contendo o toxoide tetânico, recomenda-se um total de cinco doses adequadamente em intervalos designados, duas doses com intervalo de um mês entre elas, a terceira dose, ao menos, seis meses mais tarde, e depois uma dose em cada gravidez posterior (ou em intervalos de ao menos um ano), para um total de cinco doses ${ }^{10-11}$.

No contexto da sala de vacina, um dos grandes desafios é fazer com que o paciente adulto preserve seu cartão de vacina, que é um documento que comprova o histórico vacinal e deve ser guardado junto com os documentos de identificação pessoal, a fim de evitar vacinação desnecessária, aumentando o risco de eventos adversos e custos nos programas de imunização. Estudos demonstram que existem níveis duradouros de imunidade protetora antitoxina na maioria dos vacinados ${ }^{8-9,12}$, por isso é necessário a apresentação do cartão de vacina para 
dar continuidade ao esquema de vacinação iniciado anteriormente. Ainda, a obtenção de registros de vacinas administradas após a infância, incluindo dose de reforço, tal como o toxoide tetânico, administrado nas gestantes, é ainda mais difícil ${ }^{11}$.

O PAI da República Dominicana tem apresentado um avanço extraordinário em relação as coberturas vacinais para todos os imunobiológicos, próximo a 92\%, protegendo mais de dois milhões de pessoas contra a difteria e o tétano, com prioridade para as mulheres em idade fértil e grávidas ${ }^{4}$. Apesar de apresentar uma tendência estável a nível nacional, observa-se uma heterogeneidade de cobertura entre os municípios: cobertura menor de $80 \%$ em $40 \%$ dos municípios e superior a $95 \%$ em $30 \%{ }^{4}$. Para a Organização Mundial de Saúde é preciso redobrar os esforços para chegar aos grupos mais vulneráveis, principalmente os entornos urbanos desfavorecidos, e regiões isoladas por conflitos, a fim de melhorar as coberturas vacinais e diminuir as doenças imunopreveníveis ${ }^{13}$.

Em Santiago de los Caballeros/República Dominicana pressupõe que existe uma heterogeneidade de cobertura vacinal de dT entre as gestantes. Isto pode estar relacionada à perda do cartão de vacina e ao grande número de gestantes imigrantes, principalmente do Haiti. Nesse sentido, pergunta-se: as gestantes moradoras em Santiago de los Caballeros/ República Dominicana estão adequadamente vacinadas contra a difteria e o tétano? Em uma cooperação entre uma universidade do Brasil e a Organização dos Estados Americanos - OEA, para realização de mestrado, por meio da internacionalização, foi solicitado a realização de uma investigação a fim de analisar a administração de dose de dT durante a gravidez em Santiago de los Caballeros.

Assim, este estudo tem como objetivo analisar o histórico vacinal da vacina dT e os fatores relacionados à vacinação deste imunobiológico em grávidas de Santiago de los Caballeros/ República Dominicana em 2016.

\section{Método}

\section{Tipo de estudo}

Estudo transversal, descritivo e analítico, realizado com as puérperas da maternidade do Hospital José María Cabral e Báez (HJMCB) da província de Santiago de los Caballeros/República Dominicana em 2016.

\section{Local do estudo}

O HJMCB é um hospital geral de terceiro (III) nível de atenção, público e de referência para atenção prénatal. A maternidade do hospital tem 7 quartos de enfermaria, totalizando 40 leitos. Quatro salas de parto com 24 leitos e o serviço de neonatologia que inclui a Unidade de Cuidados Intensivos (UCI) para os recémnascidos de risco. É referência para toda a cidade de Santiago, região do norte do país com média de 479 partos por mês.

\section{Coleta de dados}

Considerando uma população de 10328 nascimentos no ano de 2014 distribuídos nas 16 maternidades da cidade de Santiago, para uma prevalência geral de 50\%, um nível de confiança de $95 \%$ e uma margem de erro de $5 \%$, e um efeito de desenho de 2 , foi estimada uma amostra de aproximadamente 741 gestantes.

Entretanto, devido à limitação do tempo para a coleta de dados, elegeu-se uma amostra de conveniência que incluiu todas as mulheres cujos partos foram assistidos no HJMCB no período de 05 de janeiro a 20 de março de 2016, todas as semanas de segundas-feiras a sextas-feiras. A coleta realizada nesse período justifica-se pela disponibilidade da pesquisadora em visita ao País. Todas as puérperas, independentemente do resultado do parto (óbito fetal ou nascido vivo), foram convidadas a participar do estudo durante os dias de hospitalização pósparto. Foram excluídas todas as mulheres atendidas por aborto e as puérperas que não aceitaram participar do estudo. As entrevistas e os dados foram coletados pela pesquisadora, a fim de se obter uma padronização na estratégia.

Realizou-se uma entrevista durante a permanência hospitalar pós-parto por meio de um questionário com questões relacionadas aos aspectos 
sociodemográficos, obstétricos e dados sobre vacinação da puérpera. Além do questionário, também foi utilizado como instrumento de coleta de dados o cartão de vacinação das mulheres. Quando a mulher não apresentava o cartão de vacina, solicitava-se a ela ou ao responsável, no caso das adolescentes grávidas, informações sobre os antecedentes de vacinação de dT verbalmente, com o registro no questionário como informação verbal.

O questionário foi elaborado e consensuado entre os pesquisadores da universidade do Brasil e da Universidade de Santiago de los Caballeros (RP). Foi realizado pré-teste com 17 puérperas do HJMCB para adequar as questões do instrumento de investigação. Além do pré-teste visitou-se uma sala de vacina de uma Unidade de Atenção Primária (UNAP) para verificar manuais de vacinação, notas técnicas, livros de doses registradas. Foram entrevistadas duas enfermeiras da UNAP, sobre o calendário de vacina das gestantes e dois obstetras, sobre a vacinação contra o tétano durante a gestação. Essas informações foram fundamentais para a adequação do instrumento. Após a entrevista com as enfermeiras, a variável renda foi substituída por quantas pessoas moram na casa e quantas pessoas trabalham na casa.

A variável desfecho do estudo foi a administração de dose de dT durante a gestação, categorizada em uma dose, duas (2) doses ou mais. As variáveis independentes foram; idade (15 ou menos, 16 a 19, 20 a 34, 35 ou mais); escolaridade da mãe (8 anos ou menos, 9 a 11 anos, 12 anos ou mais); estado civil (casada união livre, solteira, separada/divorciada); nacionalidade (dominicana, haitiana ou outro), número de filhos vivos (1, 2 ou mais); número de consultas de pré-natal (1 a 3, 4 a 6, 6 ou mais); idade gestacional do parto (22 a 36 semanas, 37 a 42 semanas); indicação de vacina durante o pré-natal (sim ou não); apresentação de cartão de vacina (sim ou não); profissional que indicou a vacina (médico, enfermeira, médico e enfermeira, outros). As variáveis quantas pessoas moram na casa (vive sozinha, 2 pessoas, 3 a 5, 6 ou mais pessoas); quantas pessoas trabalham na casa (1, 2 a 3, 4 ou mais, nenhuma trabalha); religião (católica, evangélica, sem religião); tipo de parto (normal ou cesárea); quando iniciou o pré-natal $\left(1^{\circ}, 2^{\circ}\right.$ ou $3^{\circ}$ trimestre de gestação); lugar que realizou o pré-natal (UNAP, hospital de $2^{\circ}$ nível, hospital de $3^{\circ}$ nível, clínica e não fez pré-natal); estado de vacinação (completo, incompleto, doses atrasadas, doses agendadas); foram utilizadas apenas para a análise descritiva da população do estudo.

\section{Análise dos dados}

Considerou-se o estado de vacinação de dT completo, quando o cartão continha o registro de três ou mais doses de vacina de difteria e tétano (tríplice bacteriana, tetravalente, pentavalente, dT). As mulheres com antecedentes de vacinação incompleta eram as que tinham menos de três registros de vacinas que continham o componente dT em cartão, a situação de doses agendadas e atrasadas foram aquelas que apresentaram cartão com dose agendadas ou atrasadas independentemente do número. Para a análise do estado de vacinação, foi considerado somente o cartão de vacina. Para a variável dose administrada durante a gravidez, foi considerado tanto o cartão de vacina como o relato verbal da mulher.

O número de filhos foi categorizado em um filho e dois ou mais por considerar que normalmente a mulher recebe a vacina de dT na primeira gestação, tendo em conta que a maioria dos adultos não mantém atualizado seu histórico vacinal. A partir do segundo filho, de acordo com o intervalo entre os partos, não é necessária a vacinação com dT, exceto nos programas de imunizações com a introdução da dTpa.

Para a tabulação e análise dos dados, utilizou-se o programa Epi Data e STATA versão 12.0, com dupla entrada para eliminar possíveis erros de digitação. A análise descritiva contemplou o cálculo das distribuições de frequências absolutas e relativas. Para avaliar os fatores associados à administração de dose de dT durante a gestação em nível bivariado, realizou-se a Regressão Simples de Poisson com variâncias robustas. Para a construção do modelo multivariado final, também com a 
técnica de Regressão de Poisson com variâncias robustas, utilizou-se, como critérios para inclusão das variáveis, o valor $\mathrm{p} \leq 0,20$ obtido na análise bivariada. Assim, Razões de Prevalência (RP) ajustadas e seus respectivos Intervalos de Confiança de 95\% (IC 95\%) foram calculados. Para a verificação de ajuste do modelo final, realizou-se o teste de Hosmer \& Lemeshow. Para todas as análises, o nível de significância estatística estabelecido foi de $5 \%(\mathrm{p}<0,05)$.

O Projeto foi aprovado pelo comitê de investigações do Hospital José María Cabral e Báez e pelo Comitê de Ética em Pesquisa da Universidade Federal de São João del Rei Campus Centro Oeste sobre Parecer $n^{\circ}$ 1.234.830. As puérperas interessadas assinaram o termo de consentimento livre e esclarecido e foram entrevistadas na própria maternidade após o parto. Foi assinado o Termo de assentimento pelo responsável da adolescente e pela puérpera.

\section{Resultados}

Participaram do estudo 726 puérperas da cidade de Santiago de los Caballeros que realizaram o parto no hospital José María Cabral e Báez. A média de idade das puérperas foi de 23,1 anos (DP: $\pm 6,2$ ). Das puérperas entrevistadas $462(63,6 \%)$ apresentaram ao menos nove anos de estudo; 613 com estado civil casada união livre $(84,4 \%)$; em relação à crença religiosa constatou-se que 331 eram católicas $(45,6 \%)$ e a nacionalidade predominante obviamente era a dominicana com 498 puérperas (68,6\%). A maioria das puérperas, 566 (78\%), viviam em casas com mais de três pessoas e quando foram perguntadas sobre quantas pessoas trabalhavam para manter o sustento da casa $485(66,8 \%)$ delas responderam que somente uma pessoa trabalhava (TABELA 1). 
TABELA 1 - Perfil sociodemográfico de puérperas atendidas na maternidade de Santiago de los Caballeros, República Dominicana, 2016.

\begin{tabular}{|c|c|c|}
\hline Variáveis & $\mathbf{n}$ & $\%$ \\
\hline \multicolumn{3}{|l|}{ Idade (anos) } \\
\hline 15 ou menos & 43 & 5,9 \\
\hline $16-19$ & 208 & 28,6 \\
\hline $20-34$ & 427 & 58,8 \\
\hline 35 o mais & 48 & 6,7 \\
\hline \multicolumn{3}{|l|}{ Escolaridade (anos) } \\
\hline 8 ou menos & 192 & 26,5 \\
\hline $9-11$ & 462 & 63,6 \\
\hline 12 o mais & 72 & 9,9 \\
\hline \multicolumn{3}{|l|}{ Estado civil } \\
\hline Casada/ União livre & 613 & 84,4 \\
\hline Solteira & 105 & 14,5 \\
\hline Separada/divorciada & 8 & 1,1 \\
\hline \multicolumn{3}{|l|}{ Religião } \\
\hline Católica & 331 & 45,6 \\
\hline Evangélica & 227 & 31,3 \\
\hline Sem religião & 168 & 23,1 \\
\hline \multicolumn{3}{|l|}{ Nacionalidade } \\
\hline Dominicana & 498 & 68,6 \\
\hline Haitiana & 228 & 31,4 \\
\hline \multicolumn{3}{|l|}{ Quantas pessoas moram na casa } \\
\hline Mora sozinha & 2 & 0,2 \\
\hline 2 pessoas & 32 & 4,4 \\
\hline 3 a 5 pessoas & 566 & 78,0 \\
\hline 6 ou mais pessoas & 126 & 17,4 \\
\hline \multicolumn{3}{|l|}{ Quantas pessoas trabalham } \\
\hline 1 pessoa & 485 & 66,8 \\
\hline 2 a 3 pessoas & 146 & 20,1 \\
\hline 4 ou mais pessoas & 92 & 12,7 \\
\hline Nenhuma trabalha & 3 & 0,4 \\
\hline
\end{tabular}

A TABELA 2 apresenta o perfil obstétrico e de vacinação das puérperas e pode ser percebido que 380 $(52,3 \%)$, ou seja, mais da metade delas tinha entre 2 a 5 filhos; $505(71,5 \%)$ iniciou-se o pré-natal no primeiro trimestre de gestação. O parto foi realizado entre 37-42 semanas, com média de 38 (DP: $\pm 4,6$ ) semanas para 556 $(76,8 \%)$ delas. No presente estudo, 346 (47,7\%) das puérperas realizaram mais de seis consultas, porém um número significativo, $261(35,9 \%)$ realizou 1 a 3 consultas. Apesar do parto vaginal ser o mais utilizado, prevaleceu um alto número de cesáreas realizadas em 349 $(48,1 \%)$ das mulheres.

Na República Dominicana, as mulheres grávidas são vacinadas com a vacina dupla adulto $(\mathrm{dT})$ e vacinação anual contra a gripe. O profissional que mais indicou a vacina durante a gestação foi o médico com 503 (69,3\%) indicações. Somente 144 (19,9\%) puérperas apresentaram o cartão de vacina no momento da coleta de dados. Entre as mulheres que apresentaram o cartão de vacina foi possível identificar que a maioria, 108 (75\%) estava com doses agendadas mesmo após o parto. Somente $11(7,6 \%)$ estavam com vacina atrasada.

Quando se perguntou a respeito da quantidade de dose recebida durante a gravidez, $416(57,3 \%)$ disse que recebeu duas ou mais doses; 203 (28\%) tomaram uma dose e $85(11,7 \%)$ nenhuma dose (TABELA 2). 
TABELA 2 - Perfil obstétrico e vacinal de puérperas atendidas na maternidade de Santiago de los Caballeros, República Dominicana, 2016.

\begin{tabular}{|c|c|c|}
\hline Variáveis & $\mathbf{n}$ & $\%$ \\
\hline \multicolumn{3}{|l|}{ Número de filho vivo } \\
\hline 1 & 322 & 44,4 \\
\hline $2-5$ & 380 & 52,3 \\
\hline Mais de 5 & 24 & 3,3 \\
\hline \multicolumn{3}{|l|}{ Número de consulta PN } \\
\hline $1-3$ & 261 & 35,9 \\
\hline $4-6$ & 119 & 16,4 \\
\hline Mais de 6 & 346 & 47,7 \\
\hline \multicolumn{3}{|l|}{ Unidade que realizou $P N$} \\
\hline UNAP & 385 & 53,0 \\
\hline Hospital de $2^{\circ}$ nível & 198 & 27,3 \\
\hline Hospital de $3^{\circ}$ nível & 31 & 4,3 \\
\hline Clínica & 106 & 14,6 \\
\hline Não realizou pré-natal & 6 & 0,8 \\
\hline \multicolumn{3}{|l|}{ Trimestre que iniciou PN } \\
\hline $1^{\circ}$ trimestre & 505 & 71,5 \\
\hline $2^{\circ}$ trimestre & 175 & 24,8 \\
\hline $3^{\circ}$ trimestre & 26 & 3,7 \\
\hline \multicolumn{3}{|l|}{ Idade gestacional $^{\mathrm{a}}$} \\
\hline $22-36$ & 168 & 23,2 \\
\hline $37-42$ & 556 & 76,8 \\
\hline \multicolumn{3}{|l|}{ Tipo de parto } \\
\hline Parto normal & 377 & 51,9 \\
\hline Parto cesárea & 349 & 48,1 \\
\hline \multicolumn{3}{|l|}{ Indicação sobre a vacina durante PN } \\
\hline $\operatorname{Sim}$ & 640 & 88,2 \\
\hline Não & 86 & 11,8 \\
\hline \multicolumn{3}{|l|}{ Profissional que indicou vacina } \\
\hline Médico & 503 & 69,3 \\
\hline Enfermeiro & 111 & 15,2 \\
\hline Médico e Enfermeiro & 7 & 1 \\
\hline Outros & 20 & 2,8 \\
\hline Não recebeu indicação & 85 & 11,7 \\
\hline \multicolumn{3}{|l|}{ Doses dT na gestação } \\
\hline Nenhuma dose & 85 & 11,7 \\
\hline Não se recorda & 22 & 3,0 \\
\hline Uma dose & 203 & 28,0 \\
\hline Duas doses & 325 & 44,7 \\
\hline Três ou mais doses & 91 & 12,6 \\
\hline \multicolumn{3}{|l|}{ Estado de vacinação ${ }^{b}$} \\
\hline Completo & 18 & 12,5 \\
\hline Incompleto & 7 & 4,9 \\
\hline Doses atrasadas & 11 & 7,6 \\
\hline Doses agendadas & 108 & 75 \\
\hline
\end{tabular}

aDuas puérperas desconheciam a idade gestacional. ${ }^{\mathbf{b}}$ Avaliado somente pelo cartão de vacina. PN: pré-natal. UNAP: Unidade de Atenção Primária. dT: vacina adsorvida difteria e tétano adulto. 
Os resultados da regressão Simples de Poisson com a Razão de Prevalência (RP) bruta de dose administradas durante a gravidez, de acordo com variáveis sociodemográficas e obstétricas são apresentados na Tabela 3 .

Observou-se associação positiva entre a variável idade e doses de dT. Mães jovens possuem 1,3 vezes mais chances de ter recebido doses de dT se comparadas com

TABELA 3 - Regressão simples de Poisson para os fatores associados a doses de dT durante a gestação, Santiago de los Caballeros, República Dominicana, 2016.

\begin{tabular}{|c|c|c|c|c|}
\hline \multirow{2}{*}{ Variáveis } & \multicolumn{2}{|c|}{ Doses de dT - gestação } & \multirow{2}{*}{ RP bruta (IC 95\%) } & \multirow{2}{*}{$p^{\mathbf{a}}$} \\
\hline & 2 ou 3 & 1 & & \\
\hline \multicolumn{5}{|l|}{ Idade (anos) } \\
\hline 15 ou menos & 69,7 & 30,3 & $1,34(1,08-1,67)$ & 0,008 \\
\hline $16-19$ & 67,8 & 32,2 & $1,30(1,14-1,49)$ & $<0,001$ \\
\hline $20-34$ & 52,0 & 48,0 & referência & - \\
\hline 35 ou mais & 47,9 & 52,1 & $0,92(0,68-1,26)$ & 0,605 \\
\hline \multicolumn{5}{|l|}{ Escolaridade (anos) } \\
\hline 8 ou menos & 51,0 & 49,0 & $0,92(0,72-1,18)$ & 0,505 \\
\hline $9-11$ & 60,2 & 38,8 & $1,08(0,87-1,35)$ & 0,476 \\
\hline 12 ou mais & 55,6 & 44,4 & referência & - \\
\hline \multicolumn{5}{|l|}{ Estado civil } \\
\hline Casada/União livre & 58,7 & 41,3 & referência & - \\
\hline Solteira/Separada/Divorciada & 49,6 & 50,4 & $0,84(0,69-1,03)$ & 0,092 \\
\hline \multicolumn{5}{|l|}{ Nacionalidade } \\
\hline Dominicana & 60,0 & 40,0 & referência & - \\
\hline Haitiana & 51,3 & 48,7 & $0,85(0,74-0,99)$ & 0,034 \\
\hline \multicolumn{5}{|l|}{ Número de filho vivo } \\
\hline 1 & 68,0 & 32,0 & referência & - \\
\hline 2 ou mais & 48,8 & 51,2 & $0,72(0,63-0,81)$ & $<0,001$ \\
\hline \multicolumn{5}{|l|}{ Número de consulta PN } \\
\hline $1-3$ & 50,4 & 49,6 & $0,87(0,71-1,07)$ & 0,191 \\
\hline $4-6$ & 57,9 & 42,1 & referência & - \\
\hline Mais de 6 consultas & 59,2 & 40,8 & $1,02(0,89-1,17)$ & 0,731 \\
\hline \multicolumn{5}{|l|}{ Idade gestacional } \\
\hline $22-36$ & 54,8 & 45,2 & $0,95(0,81-1,10)$ & 0,478 \\
\hline $37-42$ & 57,9 & 42,1 & referência & - \\
\hline \multicolumn{5}{|l|}{ Cartão de vacina } \\
\hline Sim & 56,2 & 43,8 & referência & - \\
\hline Não & 57,5 & 42,5 & $1,02(0,87-1,20)$ & 0,778 \\
\hline
\end{tabular}

aRegressão Simples de Poisson. Variável dependente: 1- > 2 ou 3 doses; 0- > até 1 dose. IC: Intervalo de confiança. RP: Razão de prevalência. dT: vacina adsorvida difteria e tétano adulto. PN: pré-natal.

O resultado do modelo multivariado de Regressão de Poisson com RP ajustadas para os fatores associados à dose da vacina durante a gravidez apresentou que as mulheres solteiras/separadas divorciadas, com dois mães entre 20 e 34 anos. E ainda foi identificada associação negativa entre as variáveis de exposição, nacionalidade, número de filhos vivos e a variável desfecho, doses de dT. Ser mãe haitiana e ter 2 ou mais filhos diminuem as chances de tomar doses desnecessárias de dT (TABELA 3). 
TABELA 4 - Modelo multivariado final para os fatores associados a doses de dT durante a gestação, Santiago de los Caballeros, República Dominicana, 2016.

\begin{tabular}{|c|c|c|}
\hline Variáveis & RP ajustada (IC 95\%) & Valor $\mathbf{p}^{\mathbf{a}}$ \\
\hline \multicolumn{3}{|l|}{ Idade } \\
\hline 15 ou menos & $1,20(0,95-1,52)$ & 0,125 \\
\hline $16-19$ & $1,12(0,97-1,30)$ & 0,127 \\
\hline $20-34$ & referência & - \\
\hline 35 ou mais & $1,07(0,79-1,46)$ & 0,657 \\
\hline \multicolumn{3}{|l|}{ Escolaridade } \\
\hline 8 ou menos & $0,99(0,76-1,29)$ & 0,943 \\
\hline $9-11$ & $1,03(0,83-1,28)$ & 0,775 \\
\hline 12 ou mais & referência & - \\
\hline \multicolumn{3}{|l|}{ Estado civil } \\
\hline Casada/ União livre & referência & - \\
\hline Solteira/Separada/Divorciada & $0,81(0,68-0,98)$ & 0,026 \\
\hline \multicolumn{3}{|l|}{ Nacionalidade } \\
\hline Dominicana & referência & - \\
\hline Haitiana & $1,00(0,84-1,18)$ & 0,963 \\
\hline \multicolumn{3}{|l|}{ Número de fillho vivo } \\
\hline 1 & referência & - \\
\hline 2 ou mais & $0,80(0,69-0,92)$ & 0,002 \\
\hline \multicolumn{3}{|l|}{ Indicações sobre a vacina no pré-natal } \\
\hline Sim & referência & - \\
\hline Não & $0,24(0,14-0,40)$ & $<0,001$ \\
\hline
\end{tabular}

aRegressão Múltipla de Poisson. Variável dependente: 1- > 2 ou 3 doses; 0 - > até 1 dose; Ajustamento do modelo: Goodness of fit $=1,00$. IC: Intervalo de confiança. RP: Razão de Prevalência.

\section{Discussão}

Estudos realizados na França, Brasil e Austrália concluíram que o pré-natal é considerado como um dos fatores determinantes que influenciam a vacinação entre mulheres grávidas ${ }^{14-16}$. A análise multivariada demonstrou que a administração de duas ou mais doses de dT foi superior naquelas puérperas que receberam indicação de vacina durante o pré-natal quando comparado àquelas que não receberam. Ademais, na análise descritiva observou-se que o profissional que mais indicou a vacina foi o médico, divergindo dos resultados de Yengle $^{17}$, que identificou que a maioria das indicações de vacina para as gestantes são feitas por parteiras.

Nossos achados com respeito a esse tópico devem ser interpretados com cuidado, pois a medida da variável dose de dT durante a gravidez, a partir da análise do cartão de vacina e da informação das puérperas, identificou que independente de idade, número de filhos e histórico de vacinação prévio a mulher recebeu duas ou mais doses de dT. Esses resultados podem estar relacionados com a falta do cartão de vacina, a falta de conhecimento dos profissionais de saúde sobre a duração da imunidade contra o tétano e sobre o calendário de vacinação da gestante e deve ser explorado em futuros estudos sobre a temática.

Investigações realizadas no Brasil e Canadá identificaram que é frequente o desconhecimento do histórico de vacinação do adulto pela ausência do cartão de vacina ${ }^{18-19}$. Estudo de revisão documenta que seria importante que os profissionais de saúde orientassem às pessoas quanto à relevância dos dados contidos nos comprovantes de vacinação ${ }^{11}$. Autores concordaram que se deve ter um registro eletrônico nacional para rastrear as vacinas administradas a um indivíduo ${ }^{11,19-20}$. É importante realçar que em Santiago de los Caballeros não há um sistema informatizado de imunização o que pode levar a 
um comprometimento do acompanhamento do histórico de vacina das pessoas.

Dessa forma, sem o cartão de vacina e/ou histórico de vacinação considera-se a situação vacinal desconhecida e se administram doses desnecessárias. Considerando a implementação do PAI na década de 1970, muitas das mulheres incluídas nessa investigação, com idade inferior a 34 anos $(93,3 \%)$, devem ter sido vacinadas na infância e por consequência, precisariam de uma dose de reforço de dT. A complexidade do calendário de vacinação pode afetar a confiabilidade de uma história de vacinação verbal, por isso é necessário apresentação do cartão de vacinação ${ }^{11}$ e conhecer a durabilidade da imunidade induzida pela vacina é de fundamental importância para a tomada de decisões corretas sobre as indicações de doses de reforço ${ }^{12}$.

O nível de imunidade induzida por um ciclo de três dose de toxoide é alto e duradouro. Após a terceira dose, cada dose adicional com um intervalo de pelo menos um ano aumenta o nível de antitoxina tetânica prolongando a duração da imunidade ${ }^{8,21}$. A imunidade terá uma duração de dez anos após a quarta dose e pelo menos vinte anos após a quinta dose ${ }^{21-22}$ quiçá por toda a vida ${ }^{4}$. Na Coréia, um estudo revelou que três doses da vacina dT conferiram $100 \%$ de proteção contra o tétano, em indivíduos com mais de 40 anos, além de garantir imunidade protetora com longo prazo, em 94\% dos indivíduos ${ }^{8}$.

As vacinas pertencem a um dos grupos de produtos biológicos com excelente perfil de segurança, todavia não são isentas de eventos adversos. $\mathrm{Na}$ Inglaterra $^{23}$ e Estados Unidos ${ }^{24}$, foram identificados erros relacionados à avaliação incorreta do calendário de imunização e história de vacinação de paciente resultando em doses extras de vacina com diminuição de intervalo recomendado entre as doses ${ }^{23}$.

Um estudo de coorte retrospectivo para examinar o risco de reações locais após a administração da vacina dT encontrou que o risco aumenta com número de doses prévias de vacinas. Observou-se uma associação estatisticamente significativa entre o recebimento da vacina, que contém o componente do tétano, antes de cinco anos da vacinação anterior. O Comitê Assessor sobre Práticas de Imunização recomenda uma faixa mínima de 10 anos para a administração rotineira da vacina $\mathrm{dT}$, exceto na profilaxia de feridas sujas, ou gravidez, sem história prévia de dT nos últimos cinco $\operatorname{anos}^{25}$

Uma das limitações deste estudo é que somente 19,8\% das puérperas apresentaram o cartão de vacina no momento da entrevista realizada na maternidade, e o fato de verificar a vacinação baseada também em relato pode levar à possibilidade de viés de memória, visto que algumas puérperas podem ter esquecido ou ignorado dados. Outra limitação é a generalização dos resultados que deve ser vista com cuidado, já que se trata de uma população de usuários de um serviço público de saúde e não de uma mostra aleatória de base populacional. Entretanto, o estudo é importante porque mostra deficiência nos processos relacionados à vacinação em Santiago de los Caballeros. Isso leva a pensar na necessidade de criação de políticas de educação permanente para os profissionais de saúde quanto ao manejo de vacinas, além da informatização das salas de vacinação, de forma a manter o registro de vacinação atualizado, evitando doses desnecessárias de vacinas e risco de eventos adversos pós vacinação.

Assim, com base nos achados deste estudo e considerando a importancia da vacinação segura são necessárias ações de educação em saúde com os profissionais que atendem as gestantes e de discussão sobre a melhoria dos registros de vacinação e a conscientização da população sobre a importância do cartão de vacinação.

\section{Conclusão}

Houve uma administração de duas ou mais doses de dT na maioria das gestantes. Ser solteira/separada divorciada, com dois filhos ou mais e que não receberam orientação sobre a vacina durante a gestação foram considerados como fatores de proteção para a administração de duas ou mais doses de vacina de dT. A 
administração de duas ou mais doses de dT durante a gravidez pode estar relacionada à ausência de história de vacinação, o que aumenta o risco de receber doses desnecessárias de dT. Esse fato pode acarretar exposição a eventos adversos pós-vacinação e aumentar os custos do PAI.

\section{Declaração de conflitos de interesse}

Os autores declaram não haver conflitos de interesse.

\section{Referências}

1. Carteiro DMH, Sousa LMR, Caldeira SMA. Clinical indicators of sexual dysfunction in pregnant women: integrative literature review. Rev Bras Enferm. [Internet] 2016 [consultado em 9 de outubro de 2016]; 69(1):153-61. Disponível em: http://www.scielo.br/pdf/reben/v69n1/en_0034-7167reben-69-01-0165.pdf

\section{Swamy GK, Heine RP. Vaccinations for}

Pregnant Women. Obstet Gynecol. [Internet] 2015 [consultado em 15 de junho de 2016]; 125(1): 212-26. Disponível em: https://www.ncbi.nlm.nih.gov/pubmed/25560127 3. Rasmussen AS, Watson AK, Kennedy ED, Broder KR, Jamieson DJ. Vaccines and pregnancy: past, present, and future. Semin Fetal Neonatal Med. [Internet] 2013 [consultado em 15 de junho de 2016]; 19(3):161-69. Disponível em:

http://www.sfnmjournal.com/article/S1744$165 \times(13) 00123-6 / p d f$

4. República Dominicana. Secretaria de Estado de Salud Pública y Asistencia Social Sub- Secretaria de Salud Colectiva. Manual de Procedimientos Técnicos Sobre las Normas del Programa Ampliado de Inmunización. 2008.

5. Sawyer M, Liang JL, Messonnier N, Clark TA. Updated recommendations for use of tetanus toxoid, reduced diphtheria toxoid and acellular pertussis vaccine (Tdap) in pregnant women-Advisory

Committee on Immunization Practices (ACIP), 2012.

MMWR Morb Mortal Wkly Rep. [Internet] 2013

[consultado em 9 de junho de 2016]; 62(7)131-5.

Disponível em:

https://www.cdc.gov/mmwr/preview/mmwrhtml/mm620 7a4.htm

6. Housey M, Zhang F, Miller C, Lyon-Callo S, McFadden J, Garcia E. Vaccination with Tetanus,

Diphtheria, and Acellular Pertussis Vaccine of Pregnant Women Enrolled in Medicaid - Michigan, 2011-2013. MMWR Morb Mortal Wkly Rep. [Internet] 2014 [consultado em 15 de junho de 2016]; 63(38):83942. Disponível em:

https://www.cdc.gov/mmwr/preview/mmwrhtml/mm633 8a4.htm

7. United Kingdom. Department of Health

Government. Pregnant women to be offered whooping cough vaccination; 2012 [consultado em 4 de junho de 2015]. Disponível em:

https://www.gov.uk/government/uploads/system/uploads lattachment_data/file/212947/CMO-Pertussis-27-092012-FINAL.pdf

8. Choi Choo EJ, Huh A, Choi S, Eom JS, Lee JS, Park SH, Kang JH. Immunogenicity and safety of diphtheria-tetanus vaccine in adults. $\mathrm{J}$ Korean Med Sci. [Internet] 2010 [consultado em 5 de outubro de 2015]; 25(12):1727-32. Disponível em: https://www.ncbi.nlm.nih.gov/pubmed/21165286 9. Trucchi C, Zoppi G. Decennial diphtheriatetanus adult boosters: are they really necessary? J Prev Med Hyg. [Internet] 2015 [consultado em 15 de outubro de 2016]; 56(1):E44-E48. Disponível em: https://www.ncbi.nlm.nih.gov/pmc/articles/PMC471834 4/

10. Demicheli V, Barale A, Rivetti A. Vaccines for women to prevent neonatal tetanus Review. Cochrane Database Syst Rev. [Internet] 2015 [consultado em 15 de junho de 2015]; (5):1-3. Disponível em:

http://onlinelibrary.wiley.com/doi/10.1002/14651858.CD 002959.pub4/pdf/abstract

11. Cutts FT, Izurieta HS, Rhoda DA. Measuring 
Coverage in MNCH : Design, Implementation, and Interpretation Challenges Associated with Tracking Vaccination Coverage Using Household Surveys. Plos Med. [Internet] 2013 [consultado em 15 de janeiro de 2017]; 10(5):1-11. Disponível em: https://www.ncbi.nlm.nih.gov/pubmed/23667334 12. Hammarlund E, Thomas A, Poore EA, Amanna IJ, Rynko AE, Mori M, Chen Z, Slifka, MK. Durability of Vaccine-Induced Immunity Against Tetanus and Diphtheria Toxins : A Cross-sectional Analysis. Clin Infect Dis. [Internet] 2016 [consultado em 15 de janeiro de 2017]; 62(9):1111-18. Disponível em:

https://academic.oup.com/cid/article/62/9/1111/1745278/ Durability-of-Vaccine-Induced-Immunity-Against

13. World Health Organization. Maternal and neonatal tetanus elimination. 2019. [consultado em 9 de julho de 2019]. Disponível em:

https://www.who.int/immunization/diseases/MNTE_initi $\underline{\text { ative/en/ }}$

14. Blondel B, Mahjoub N, Drewniak N, Launay O, Goffinet $F$. Failure of the vaccination campaign against $\mathrm{A}(\mathrm{H1} 1 \mathrm{N1})$ influenza in pregnant women in France: results from a national survey. Vaccine. [Internet] 2012 [consultado em 4 de junho de 2015]; 30(38):5661-65. Disponível em:

https://www.ncbi.nlm.nih.gov/pubmed/22781306

15. Domingues RMSM, Hartz ZMA, Dias MAB, Leal MC. Avaliação da adequação da assistência prénatal na rede SUS do Município do Rio de Janeiro, Brasil. Cad de Saúde Pública. 2012 [Internet] [consultado el 4 de junio de 2016]; 28(3):425-37.

Disponível em: en: http://www.scielo.br/pdf/csp/v28n3/03.pdf

16. Maher L, Hope K, Torvaldsen S, Lawrence G, Dawson A, Wiley K, Thomson D, Hayen A, Conaty S. Influenza vaccination during pregnancy: coverage rates and influencing factors in two urban districts in Sydney. Vaccine. [Internet] 2013 [consultado em 7 de junho de 2016]; 31(47):5557-64. Disponível em: http://ssu.ac.ir/cms/fileadmin/user_upload/Moavenatha/ MBehdashti/Pishgiri_Bimariha/anf3/49.pdf

17. Yengle LMV, Martí MC, Roura LC, Pendás
JAR, Gómez XMG, Pérez EH, Rafart JV. Vacunación antigripal en gestantes. Cobertura vacunal y conocimientos y prácticas de los obstetras. Med Clin. [Internet] 2010 [consultado em 4 de junho de 2016]; 134(4):146-51. Disponível em: http://www.elsevier.es/es-revista-medicina-clinica-2articulo-vacunacion-antigripal-gestantes-coberturavacunal-S0025775309014006

18. Francisco PMSB, Senicato C, Donalisio MR, Barros MBA. Vacinação contra rubéola em mulheres em idade reprodutiva no Município de Campinas, São Paulo, Brasil. Cad de Saúde Pública. [Internet] 2013 [consultado em 4 de junho de 2016]; 29(3):579-88. Disponível em:

http://www.scielo.br/pdf/csp/v29n3/a15v29n3.pdf 19. Macdougall DM, Halperin BA, MacKinnonCameron D, Li L, McNeil AS, Halperin SA. The challenge of vaccinating adults: attitudes and beliefs of the Canadian public and healthcare providers.

BMJ Open. [Internet] 2015 [consultado em 7 de junho de 2016];5 (9):1-12. Disponível em:

http://bmjopen.bmj.com/content/bmjopen/5/9/e009062.fu 11.pdf

20. Oliveira TR, Costa FMR. Desenvolvimento de aplicativo móvel de referência sobre vacinação no Brasil. J Health Inform. [Internet] 2012 [consultado em 15 de janeiro de 2017]; 4(1):23-7. Disponível em: http://www.jhi-sbis.saude.ws/ojs-jhi/index.php/jhi$\underline{\text { sbis/article/view/161/109 }}$

21. World Health Organization (WHO). The immunological basis for immunization series. Module 3: tetanus. Genebra : WHO; 2006. Disponível em: http://www.who.int/immunization/documents/immunolo gical_basis_series/en/

22. Ölander RM, Auranen K, Härkänen T, Leino T. High tetanus and diphtheria antitoxin concentrations in Finnish adults - Time for new booster recommendations? Vaccine. [Internet] 2009 [consultado em 15 de junho de 2016]; 27(39):5295-98. Disponível em: https://www.ncbi.nlm.nih.gov/pubmed/19596410 23. Lang S, Ford KJ, John T, Pollard AJ, Mccarthy 51 | P á g i n a 
ND. Immunisation errors reported to a vaccine advice service: intelligence to improve practice. Qual Prim Care. [Internet] 2014 [consultado em 15 de junho de 2016]; 22(3):139-46. Disponível em: https://www.ncbi.nlm.nih.gov/pubmed/24865341

24. Hibbs BF, Moro PL, Lewis P, Miller ER,

Shimabukuro TT. Vaccination errors reported to the Vaccine Adverse Event Reporting System, (VAERS)

United States, 2000-2013. Vaccine. [Internet] 2015

[consultado em 9 de junho de 2015]; 33(28):3171-78.

Disponível em:

https://www.sciencedirect.com/science/article/pii/S0264

410X15006209?via\%3Dihub

25. Jackson La, Yu O, Nelson J, Belongia EA,

Hambidge SJ, Baxter R, Naleway A, Nordin J, Baggs J, Iskander J. Risk of medically attended local reactions following diphtheria toxoid containing vaccines in adolescents and young adults: a Vaccine Safety

Datalink study. Vaccine. [Internet] 2009 [consultado em 9 de junho de 2015]; 27(36):4912-16. Disponível em: https://www.ncbi.nlm.nih.gov/pubmed/19567245 